\title{
INFLUENCIA DEL NIVEL DE CONOCIMIENTO SOBRE SEXUALIDAD EN LOS TIPOS DE ACTITUD FRENTE A CONTENIDOS SEXUALES ENSEÑADOS EN EL ÁREA DE PERSONA, FAMILIA Y RELACIONES HUMANAS DEL NIVEL SECUNDARIO (2014)*
}

\begin{tabular}{c}
\hline Luisa Vivian Rivera Bravo ${ }^{* *}$ \\
Universidad de Ciencias y Humanidades \\
lrivera@uch.edu.pe
\end{tabular}

Fecha de recepción: agosto de 2019 Fecha de aceptación: diciembre de 2019

ReSUMEN: El presente trabajo de investigación es un estudio de la realidad educativa peruana. Precisamente se trata de la labor que cumplen los docentes en ejercicio que tienen a cargo el área de Persona, Familia y Relaciones Humanas (PFRRHH), desde donde se aborda temas de sexualidad. Para ello, se han escogido en el estudio las instituciones educativas 6080 Rosa de América y 7236 Max Uhle, pertenecientes a la UGEL N. 01.

\footnotetext{
* Este texto forma parte de la tesis de la autora para obtener el grado de magíster en Docencia Universitaria por la Universidad Nacional Mayor de San Marcos.

** Luisa Vivian Rivera Bravo es bachiller (2009) y licenciada (2009) en Educación, en la especialidad de Ciencias Histórico Sociales, por el Instituto Pedagógico Nacional Monterrico. Tiene, además, un diplomado en Neurociencias y Andragogía (2012) por la Universidad Enrique Guzmán y Valle. Ejerce la docencia desde el año 2009 en diversas instituciones educativas de diferentes niveles tanto públicas como privadas. Actualmente es docente del área Desarrollo Personal y Ciudadanía en una institución pública y del Programa de Extensión Educativa de la Universidad de Ciencias y Humanidades (Los Olivos).
} 
Las labores que han desempeñado los docentes de ambas instituciones educativas nos ponen en alerta sobre cuál es el nivel de preparación del ejercicio docente en el desarrollo de las clases, puesto que se evidencia algunas carencias en el nivel de conocimiento de contenidos y tipos de actitud respecto a la sexualidad.

Para una mejor organización, el análisis se dividirá en cuatro momentos. El primero corresponde a una introducción, donde se centra todo lo referente a su abordaje desde varias esferas de la sociedad peruana. El segundo contiene definiciones del estudio que son indispensable para entender la situación. La tercera parte corresponde a la forma del trabajo realizado. Finalmente, se presenta de manera general las aportaciones más significativas del trabajo. Así, mediante el presente trabajo, se busca dar a conocer la realidad educativa de los docentes en relación al manejo de contenidos sexuales y los tipos de actitud que asumen ante ellos, sabiendo que estamos en una sociedad con altos signos de violencia de género y que es necesario que los actores educativos comprendan bien su función dentro de las aulas de clase.

Palabras Clave: Nivel de Conocimiento y Tipo de Actitud sobre sexualidad de los docentes. Contenidos sexuales en el área de Persona, Familia y Relaciones Humanas enseñados por los docentes.

INFLUENCE OF THE LEVEL OF KNOWLEDGE ON SEXUALITY IN THE TYPES OF ATTITUDE AGAINST SEXUAL CONTENTS TAUGHT IN THE AREA OF PERSON, FAMILY AND HUMAN RELATIONS OF THE SECONDARY LEVEL (2014) 
INFLUENCIA DEL NIVEL DE CONOCIMIENTO SOBRE SEXUALIDAD EN LOS TIPOS

DE ACTITUD FRENTE A CONTENIDOS SEXUALES ENSEÑADOS EN EL ÁREA

DE PERSONA, FAMILIA Y RELACIONES HUMANAS DEL NIVEL SECUNDARIO (2014)

Aвstract: This research work is a study of the Peruvian educational reality, regarding the work carried out by teachers in charge of the area of Person, Family and Human Relations (PFRRHH), from which issues of sexuality, being the I.E under study: 6080 Rosa de América and I.E. 7236 Max Uhle, belonging to Ugel 01.

The work performed by teachers in educational institutions, will alert us to the level of preparation of the teaching exercise in the development of the classes, since it shows some lacks in the level of knowledge of content and types of attitude regarding sexuality.

For a better organization, it is analyzed from four moments. The first corresponds to an introduction, where everything related to its approach is focused from differents spheres of Peruvian society. The second contains definitions of the study that are essential to understand the situation. The third part corresponds to the form of the work performed. Finally, it presents in a general way the most significant contributions of the work. Through this, it seeks to publicize the educational reality of teachers in relation to the management of sexual content and the types of attitude they assume before them, knowing that we are in a society with high signs of gender violence and that it is necessary that the actors Educational well understand their role in classrooms.

KeYwords: Level of Knowledge and Type of Attitude about sexuality of teachers. Sexual content in the area of Person, Family and Human Relations taught by teachers. 


\section{Introducción}

$\mathrm{E}$

n la actualidad, el tema de la sexualidad ha cobrado interés, pese a que existen dificultades, controversias y obstáculos alrededor de esta temática, ya sea dentro del ámbito familiar y escolar, pues existen tabúes y miedos en relación a ella, razón por la cual, muchas veces se prefiere dejar de tratar y, caso contrario, salen a flote creencias, prejuicios y estereotipos con lo cual se va perdiendo objetividad y propiciando el no hablarse del tema, con el fin de no polemizar o quedar en ridículo. Cabe resaltar que, muchas veces, la comunicación resulta inadecuada e insuficiente dentro de los hogares y en los espacios educativos. A pesar de vivir en la sociedad del conocimiento, aún tenemos barreras que van más allá de la lógica y la razón.

En este marco, la relación entre las personas se da de manera superficial y muchas veces distorsionada, debido a la falta de valores y prevalencia del individualismo como forma de vida. Las formas de pensamiento en nuestro país en torno a la sexualidad evolucionan de manera lenta y esto no permite a las personas superar numerosos prejuicios y tabúes que impiden el crecimiento saludable de la población, siendo el más vulnerable ante esta situación el sector que comprende niños y adolescentes en desarrollo.

La vivencia de la sexualidad es un proceso de interacción que comprende el crecimiento integral del hombre en su relación con el otro, la misma que se inserta en un proceso educativo que muchas veces no permite desarrollar al estudiante en su integralidad.

Los problemas sociales de índole sexual que enfrentan niños y adolescentes en la actualidad, tales como las relaciones sexuales prematuras, el embarazo adolescente, el contagio de infecciones de transmisión sexual 
INFLUENCIA DEL NIVEL DE CONOCIMIENTO SOBRE SEXUALIDAD EN LOS TIPOS

DE ACTITUD FRENTE A CONTENIDOS SEXUALES ENSEÑADOS EN EL ÁREA

DE PERSONA, FAMILIA Y RELACIONES HUMANAS DEL NIVEL SECUNDARIO (2014)

y VIH-SIDA, se han vuelto riesgos sexuales que ponen en una constante amenaza a los estudiantes. Hay que recordar que la familia es el primer agente de socialización y de formación de valores, teniendo el deber de orientar a sus hijas e hijos en relación a estos temas.

La institución educativa, consciente de su rol dentro de este contexto, ha visto conveniente insertar como tema transversal la Educación Sexual que se define, según el Manual del Docente PFRRHH (2009), como "El conjunto de aprendizajes producto de acciones directas o indirectas, deliberadas o no, conscientes o no, ejercidas sobre un individuo a lo largo de su desarrollo, que le permiten situarse en relación a la sexualidad, en general, y a su vida sexual, en particular" (p. 31). Así, se considera importante resaltar las implicancias de abordar el tema de la sexualidad desde la labor docente, ya que el área de PFRRHH contempla a la sexualidad como una temática básica e importante para el desarrollo de los adolescentes.

La vivencia de la sexualidad se da como un proceso de interacción que permite el desarrollo integral del ser humano. Según Bernabé Sarabia (2002), en el ámbito educativo este proceso se acentúa debido a que, en la escuela, el docente participa activamente en la formación de los estudiantes brindando información, fomentando la reflexión y la toma de conciencia en la vivencia de la sexualidad.

En este sentido, es primordial la preparación de los docentes, pues la educación no solo se limita a la transmisión de conocimientos, sino también a la formación en valores que contribuyan al desarrollo integral de las personas. Cuando un docente tiene una base sólida y firme en educación sexual, encamina el desarrollo de una sexualidad madura en sus estudiantes, evidenciando confianza personal y equilibrio emocional en la relación con ellos. 


\section{Definiciones de Estudio}

n relación a los indicadores de estudio, nivel de conocimiento y tipo
de actitud sobre la sexualidad, se ha distribuido los contenidos en
relación a los indicadores como se ve en la Tabla 1 .

Tabla 1

Contenidos del área de Persona, Familia y Relaciones Humanas y el bloque temático propuesto

\section{Contenidos del Área de Persona, Familia y Relaciones Humanas \\ Bloque temático propuesto}

- La sexualidad.

- Las concepciones sobre la sexualidad.

- Derechos sexuales y reproductivos.

Nociones básicas.

- Riesgos en la sexualidad.

- Factores protectores en la sexualidad.

- Características de varones y mujeres.

- Conducta de varones y mujeres en la Características sexuales en la adolescencia. adolescencia.

- Las manifestaciones de la sexualidad. 
INFLUENCIA DEL NIVEL DE CONOCIMIENTO SOBRE SEXUALIDAD EN LOS TIPOS

DE ACTITUD FRENTE A CONTENIDOS SEXUALES ENSEÑADOS EN EL ÁREA

DE PERSONA, FAMILIA Y RELACIONES HUMANAS DEL NIVEL SECUNDARIO (2014)

- Creencias acerca de la conducta de varones y mujeres.

- Lenguaje sexista.

- Valores y sexualidad.

Valores, creencias y prejuicios sobre sexualidad.

- Mitos y/o estereotipos sobre la sexualidad.

- Las relaciones entre grupos de varones y mujeres.

- Cambios del desarrollo en las relaciones con los pares.

Relaciones interpersonales.

- El enamoramiento.

- Relación de pareja.

Como dice el Ministerio de Educación (2009), en su Diseño Curricular Nacional los contenidos mostrados están desarrollados en el Manual del Docente de PFRRHH, el mismo que ha sido entregado gratuitamente por el gobierno nacional a las Instituciones Educativas de nuestro país.

Los bloques temáticos utilizados sirven para categorizar el nivel de conocimiento sobre sexualidad. Las "nociones básicas" hacen referencia a la sexualidad como un conjunto, forma parte de una experiencia que se desarrolla a lo largo de la vida y está presente en todas sus etapas. Son las vivencias, expectativas, características físicas e intereses; es decir, comprende íntegramente todo lo que somos. El nuevo enfoque de salud sexual enfatiza el sentido positivo y enriquecedor de la sexualidad en la vida de las personas. Por ello, es necesario garantizar el bienestar físico, psicológico y social de la persona, en relación a la vivencia de su sexualidad. 
Las "Características sexuales en la adolescencia", del Manual del Docente del área de PFRRHH (2009), refiere que "la adolescencia está caracterizada por transformaciones físicas, psíquicas y sociales, tales como la maduración sexual y el desarrollo cognitivo; lo que trae consigo cambios mentales, sociales y emocionales, así como nuevas experiencias, responsabilidades y relaciones con los compañeros y compañeras" ( $\mathrm{p}$. 17). En consecuencia, se puede afirmar que los cambios que se inician con la pubertad son un proceso continuo de transformaciones biológicas y emocionales que implican la maduración del adolescente, el mismo que atraviesa un período de su vida particularmente intenso en el cual construirá su propio camino y tomará conciencia de sí mismo y de los cambios que se dan en su cuerpo, además de los sentimientos y pensamientos que estos generan.

Los "Valores, creencias y prejuicios de la sexualidad", tienen como cimiento a los estereotipos sociales, las creencias acerca de la sexualidad y los valores de la sexualidad que están fuertemente enraizados en nuestra cultura, incluso de manera inconsciente. El manual del docente de PFRRHH (2009), afirma que "en nuestra cultura existen muchas ideas acerca de cómo deben ser los hombres y mujeres que corresponden a un modelo machista, es decir se basan en valores que promueven el poder de los varones sobre las mujeres" (p. 30). Se muestra, de ese modo, la existencia de ciertas conductas estereotipadas en la sociedad peruana, las cuales dificultan las relaciones entre varones y mujeres, impidiendo que sean equitativas, plenas y satisfactorias.

Las "Relaciones interpersonales" versan sobre los lazos que el adolescente empieza a establecer con sus pares como resultado de la necesidad de crear nuevas relaciones, pues ello le aporta seguridad y reconocimiento, a la vez que le brinda la posibilidad de compartir afectos, ideas, temores, 
INFLUENCIA DEL NIVEL DE CONOCIMIENTO SOBRE SEXUALIDAD EN LOS TIPOS

DE ACTITUD FRENTE A CONTENIDOS SEXUALES ENSEÑADOS EN EL ÁREA

DE PERSONA, FAMILIA Y RELACIONES HUMANAS DEL NIVEL SECUNDARIO (2014)

gustos, entre otros. Mucha de la energía que el adolescente posee se centrará en sentirse integrado y aceptado por el grupo; siendo este el lugar donde podrá brindar sus opiniones y ensayar el uso de su libertad para la toma de sus decisiones.

Respecto a las "actitudes frente a contenidos sexuales", se ha visto conveniente estructurarlo en base a lo dicho por Bolívar Botia (1999), quien clasifica la actitud en permisiva, represiva y personalizadora. Se entiende por actitud permisiva, a la tolerancia exacerbada y pasiva ante cualquiera forma de expresión sexual que sean inadecuadas hasta pudiendo ser ofensiva, esta actitud rechaza la prohibición de todo tipo. La actitud personalizadora es la que reconoce que todos somos sexualmente importantes, aprendiendo a aceptarnos positivamente para mejorar nuestra autoestima sexual, generando confianza y permitiendo la toma de decisiones responsables. La actitud represiva es el rechazo y negación hacia las manifestaciones de la sexualidad, lo cual trae consigo muros que impiden el conocimiento de nuestro propio ser, no favoreciendo al desarrollo pleno del hombre. Se hace necesario educar las actitudes como afirma Alcántara (1992), ya que permitirá el desarrollo de compontes conductuales, cognitivos y afectivos adecuados hacia los estudiantes.

\section{Forma de Trabajo y Aportes de la Investigación}

T a forma de trabajo en el estudio tuvo como instrumentos un cuestionario y una escala tipo Likert, los que permitieron medir el nivel de conocimiento y el tipo de actitud, respectivamente. Los docentes en ejercicio, pertenecientes a las instituciones educativas evaluadas, dan cuenta que dominan contenidos relacionados a las concepciones de 
la sexualidad, derechos sexuales y reproductivos, riesgos en la sexualidad y factores protectores en la sexualidad. Sin embargo, aún hay un grupo minoritario de docentes que carece de preparación en relación a estos temas.

Respecto a la dimensión "Características sexuales en la adolescencia”, estas dan cuenta que los docentes se hallan en un nivel medio, situación preocupante preocupante sabiendo que un docente tiene que dominar estos contenidos para poder responder a las necesidades y demandas de los estudiantes.

Sobre la dimensión "Valores, creencias y prejuicios sobre sexualidad", a los docentes les falta dominar temas como las creencias acerca de la conducta de varones y mujeres, lenguaje sexista y mitos y estereotipos sobre sexualidad. Tales insuficiencias se convierten en desafío que tienen que afrontar los docentes, ya que deben lidiar con sus propios prejuicios sobre la sexualidad cuando abordan sesiones de aprendizajes.

Frente a la dimensión "Niveles de Relaciones interpersonales", los resultados de la investigación dan cuenta que la mitad de docentes presentan un nivel de conocimiento alto en relación con los contenidos de sexualidad. Gracias a ello, se demuestra que los docentes comprenden temáticas relacionadas a los grupos de varones y mujeres, cambios en el desarrollo de las relaciones entre pares, el enamoramiento y relación de pareja.

En relación a los “Tipos de actitud de los docentes del área de PFRRHH", la actitud personalizadora, que es la más adecuada para tratar temas de sexualidad, debido a que fomenta el respeto, empatía y asertividad con los estudiantes, los docentes de las instituciones educativas Rosa de América y Max Uhle se hallan en un nivel medio. El motivo radica en que no están cumpliendo de manera eficaz en las sesiones de aprendizaje, al orientar de manera adecuada a los adolescentes. 
INFLUENCIA DEL NIVEL DE CONOCIMIENTO SOBRE SEXUALIDAD EN LOS TIPOS

DE ACTITUD FRENTE A CONTENIDOS SEXUALES ENSEÑADOS EN EL ÁREA

DE PERSONA, FAMILIA Y RELACIONES HUMANAS DEL NIVEL SECUNDARIO (2014)

En cuanto a la actitud represiva, los docentes se hallan en el nivel medio cuando desarrollan las sesiones de clase. A partir de ello, se concluye que la mayoría de los docentes no asumen una actitud adecuada ni realizan una explicación eficiente ni eficaz donde prime el diálogo sobre temas de sexualidad.

Por lo hallado en este estudio es necesario que se oriente la formación docente en actitudes y valores en el ámbito de la sexualidad. De esta manera se podrá fomentar el respeto a la vida humana, la responsabilidad, la equidad, la solidaridad y la empatía en sus estudiantes. Al respecto, Ariza, Cesari y Gabriel (2000), afirman que un educador, preocupado por una educación integradora de las diversas áreas de la personalidad del chico (a) no puede olvidar que la sexualidad es el trasfondo de todo el desarrollo del alumno y que él mismo educa desde las actitudes que configuran su propia persona.

Es decir, un docente debe estar preparado para la labor de educar en sexualidad y responder a las necesidades de sus estudiantes y conocer la personalidad de los adolescentes para favorecer una educación integradora. Es necesario reforzar los conocimientos en materia de la sexualidad para que los docentes sigan siendo buenos mediadores del aprendizaje y facilitadores en relación a la misma.

La educación sexual del docente es muy importante debido a la influencia que ejerce directamente en la toma de decisiones de los alumnos y es por esta razón que todo estudiante de educación tiene una gran responsabilidad: la de ser un buen formador. Así, la formación inicial se propone el orientar una educación constructiva de estudiantes capaces de formar integralmente a sus alumnos.

Dentro de la Institución Educativa es importante señalar, como factor importante, el rol del docente debido a que su intervención en el 
proceso de desarrollo del estudiante es lograr que desarrolle competencias y capacidades para la vida. Por lo tanto, la formación del docente en este tema debe partir de un manejo adecuado de los contenidos, además de evidenciar con su vida, comportamientos saludables.

Durante la labor del docente en servicio es necesario que este posea una motivación intrínseca que le permita realizar la búsqueda de información y la autorreflexión sobre los contenidos de sexualidad relacionada al área de PFRRHH, ya que el aprendizaje de nuevos conocimientos podrá dirigir el tipo de actitud frente a estos contenidos.

Como afirma el Ministerio de Educación (2005), en el Manual de Tutoría, los docentes que están encargados del área de PFRRHH tienen una misión muy especial. Ellos no solo desarrollan temas relacionados a psicología o filosofía, sino que esta área abarca temas de sexualidad, motivo por cual es necesario que los contenidos que se proponen en el Diseño Curricular Nacional (DCN) deberían ser de conocimiento y manejo de los docentes, entendiendo que la sexualidad forma parte de la vida de todas las personas $y$, por tanto, hay que considerarla como un elemento integrador que une aspectos cognitivos, afectivos y conductuales.

Se recomienda, entonces, que las autoridades de las instituciones educativas 6080 Rosa de América y 7236 Max Uhle, de la UGEL N. 01 de Lima Metropolitana, reconozcan la necesidad urgente de mejorar el nivel de conocimiento de los contenidos de sexualidad de los docentes del área de PFRRHH del nivel secundario. Esto puede darse a través de la puesta en marcha de eventos, capacitaciones o charlas, las cuales coadyuven a la mejora de la metodología docente y el dominio de la materia acorde a la asignatura o nivel en que cada docente se desempeña. Se debe entender, 
INFLUENCIA DEL NIVEL DE CONOCIMIENTO SOBRE SEXUALIDAD EN LOS TIPOS

DE ACTITUD FRENTE A CONTENIDOS SEXUALES ENSEÑADOS EN EL ÁREA

DE PERSONA, FAMILIA Y RELACIONES HUMANAS DEL NIVEL SECUNDARIO (2014)

también, que el docente está en permanente formación y capacitación para formar integralmente al estudiante.

Se requiere que se formen a los docentes en reflexión para que así sean capaces de hacer reflexionar a sus estudiantes sobre las problemáticas sexuales que afronta la sociedad, dado que los tiene como protagonistas.

\section{Conclusiones}

L

a educación sexual, debe partir de los hogares, pero consolidándose en la escuela, por ello la institución educativa, debe seguir su rol de formación en valores teniendo como pilares a los docentes, que deben recibir el apoyo de la Unidades de Gestión de su Localidad (UGEL), así como desde el Ministerio de Educación, con sus cursos desde Perúeduca y diplomados en actualización sobre temas de sexualidad y mejora de su desempeño docente.

Por tanto, en la actualidad se hace necesario incidiren el protagonismo que tiene el docente como agente mediador de los aprendizajes, donde su labor vaya de la mano con conocimientos adecuados y actualizados sobre sexualidad y la forma de abordaje que responda a las necesidades e interés de los adolescentes de hoy. 


\section{REFERENCIAS}

Alcántara Juan, J. A. (1992). Cómo educar las actitudes. Lima, Perú: Editorial CEAC.

Ariza, C., Cesari, D., M. \& Gabriel, M. (2000). Programa integrado de pedagogía sexual en la escuela. Madrid, España: Narcea.

Bernabé Sarabia, E. (2002). Los contenidos en la Reforma enseñanzaaprendizaje de conceptos, procedimientos. Espańa: Editorial Santillana.

Bolívar Botia, A. (1999). La evaluación de valores y actitudes. Madrid, Espańa: Anaya.

Ministerio de Educación. (2005). Tutoría y orientación educativa. Lima, Perú: Autor.

Ministerio de Educación. (2009). Manual del docente del Area de Persona, Familia y Relaciones Humanas (T. 1-5). Lima, Perú: Autor.

Ministerio de Educación. (2009). Diseño Curricular Nacional de Educación Básica Regular. Lima, Perú: Autor. 